\title{
Resveratrol distinctively modulates the inflammatory profiles of immune and endothelial cells
}

\author{
Joseph Schwager*, Nathalie Richard, Franziska Widmer and Daniel Raederstorff
}

\begin{abstract}
Background: The phenolic substance resveratrol (RES) is a plant metabolite known to modulate numerous physiological functions and to exert beneficial effects as a cancer-chemopreventing agent and on neurological, hepatic, and cardiovascular systems. Since the compound affects the lifespan of yeast and flies it might be an anti-aging substance. Mechanistically, RES is involved in down regulating the inflammatory response. The pleiotropic effects of RES in cells of the immune and endothelial system were examined in this study.

Results: Murine macrophages (RAW264.7 cells), human monocytic/leukemia cells (THP-1), PBLs and HUVECs were incubated with RES and activated with inflammatory stimuli such as LPS or TNF-a. Inflammatory mediators and adhesion molecules were measured by multiplex analysis and gene expression was quantified by RT-PCR. In PBLs, which were activated with LPS, RES blunted the production of TNF-a, CCL2/MCP-1, CCL5/RANTES, CXCL8/IL-8, whereas it increased the production of IL-1 $\beta, I L-6, C C L 4 / M I P-1 \beta$ and CXCL10/IP-10. Thus, in the blood compartment chemokines attracting mainly monocytes were up-regulated by RES, while those attracting $T$ lymphocytes or neutrophils were diminished. At conditions of endothelial dysfunction (ED), RES reduced the expression of cytokines, chemokines, ICAM and GM-CSF in TNF- $a$ activated HUVECs, whereas eNOS expression was corrected to pre-ED homeostasis. In macrophages nitric oxide, PGE 2 cytokines (TNF-a, IL-1 $\beta, \mathrm{LL}-6)$ and chemokines (CCL2/MCP-1, CCL4/MIP-1 $\beta$, CCL5/RANTES, CXCL10/IP-10) were reduced by the phenolic substance.

Conclusions: RES had cell-specific and context-dependent effects, in particular on the expression of IL-1 $\beta, \mathrm{IL}-6$, $C C L 4 / M I P-1 \beta$ and CXCL10/IP-10. It enhanced cellular features that mirror increased alertness to disturbed immune homeostasis in the vascular-endothelial compartment (e.g. increased production of IL-1 $\beta$ or IL-6), whereas it blunted inflammatory mediators in macrophages and consequently chronic inflammation. We infer from the present in vitro study, that RES has unique properties in the regulation of inflammatory and immune responses, which are controlled in a complex hierarchical and temporal order.
\end{abstract}

Keywords: Chemokines, Cytokines, Endothelial dysfunction, Human umbilical vein endothelial cells, Inflammation, Nutrients, Peripheral blood leukocytes, THP-1 cells, RAW 264.7 cells, Resveratrol

\section{Background}

Numerous epidemiological studies have shown that a diet rich in fruits has beneficial effects on diseases such as cardiovascular diseases (CVD), diabetes, obesity and neurodegeneration. Low-grade inflammation is a common feature of these chronic diseases. As a consequence the production of mediators of inflammation is unbalanced

\footnotetext{
* Correspondence: jpschwager@gmail.com
DSM Nutritional Products Ltd., Department
Box 2676 Basel, CH-4002, Switzerland

DSM Nutritional Products Ltd., Department of Human Nutrition \& Health, P.O.

* Correspondence: jpschwager@gmail.con
DSM Nutritional Products Ltd., Departmer
Box 2676 Basel, CH-4002, Switzerland
}

(c) The Author(s). 2017 Open Access This article is distributed under the terms of the Creative Commons Attribution 4.0 International License (http://creativecommons.org/licenses/by/4.0/), which permits unrestricted use, distribution, and reproduction in any medium, provided you give appropriate credit to the original author(s) and the source, provide a link to the Creative Commons license, and indicate if changes were made. The Creative Commons Public Domain Dedication waiver (http://creativecommons.org/publicdomain/zero/1.0/) applies to the data made available in this article, unless otherwise stated. changes influence cell metabolism and alter tissue functions that are supposed to favor disease progression [1]. A hallmark of acute inflammation is the increased production of cytokines and chemokines. These enable and enhance inflammatory processes that support the recruitment of immune cells to the sites of inflammation and eliminate pathogens. Some mediators are further required during the resolution of inflammation [2] or in the differentiation of cells that orchestrate the resolution of inflammatory processes like alternatively activated macrophages [3]. During 
chronic inflammation a status of un-coordinated production of mediators and metabolites is perpetuated and causes tissue and organ damage. Consequently, inflammatory processes have an intrinsic dual nature during acute and chronic inflammation. Given the fact that diet contributes to health homeostasis, nutrients that favorably modulate the inflammatory status are supposed to prevent health disorders and diseases [4].

We investigated the in vitro effects of the phytoalexin resveratrol (RES) in acute and chronic inflammatory responses. In order to cover a potentially wide range of actions in different systemic contexts, we analyzed the effects of RES in three distinct cell types, i.e. human peripheral blood leukocytes (PBLs), human umbilical vein endothelial cells (HUVECs) and murine and human macrophage cells (RAW264.7 cells, THP-1 cells). RES has a broad pattern of biological activities related to metabolic diseases and ageing [5-7]. We show in this study, that RES markedly altered the production of cytokines and chemokines, which regulate the acute inflammatory response in peripheral blood, endothelial cells and macrophages. RES orchestrated the response of cells to inflammatory stimuli in a cell- and compartmentspecific way. Notably, RES differentially regulated the expression of inflammatory genes in various compartments of the systemic response to inflammation.

\section{Methods}

\section{Reagents}

Resveratrol (RES), E. coli lipopolysaccharide (LPS, serotype 055:B5) and fetal bovine serum (FBS) were from Sigma/ Aldrich (Saint-Louis, MO). Cell culture media (RPMI 1640, DMEM), 2-mercaptoethanol and non-essential amino acids (NEAA) were from Invitrogen (Carlsbad, CA). Recombinant human interleukin (IL)-1 $\beta$, interferon (IFN) $-\gamma$ and Tumor Necrosis Factor (TNF)- $\alpha$ were from PeproTech EC (London, UK).

\section{Cell culture}

Murine macrophage RAW264.7 and human PBLs have been cultured and treated with inflammatory stimuli as described $[8,9]$. Briefly, RAW264.7 cells were seeded into 12 -well or 96- well plates at 1 and $0.05 \times 10^{6}$ cells per well, respectively, for 2 days of preculture, starved in DMEM containing $0.25 \%$ FBS $18 \mathrm{~h}$ before the treatment and stimulated with LPS $(1 \mu \mathrm{g} / \mathrm{mL})$ for $4-24 \mathrm{~h}$ in phenol red-free DMEM containing 0.25\% FBS [8]. PBLs from healthy donors $\left(8 \times 10^{6}\right.$ viable cells $\left./ \mathrm{mL}\right)$ were cultured in phenol-red free RPMI 1640 (containing 0.25\% FBS, $0.1 \mathrm{mM}$ NEAA, $50 \mathrm{U} / \mathrm{mL}$ penicillin, $50 \mu \mathrm{g} / \mathrm{mL}$ streptomycin, $50 \mu \mathrm{M}$ 2-mercaptoethanol) and stimulated with LPS/INF- $\gamma(100 \mathrm{ng} / \mathrm{mL}, 20 \mathrm{U} / \mathrm{mL})$ with graded amounts of test substances (i.e. series of 1.56 to $50 \mu \mathrm{M}$, prepared in two-fold dilution steps from $50 \mu \mathrm{M}$ solution). RES was added to cultures shortly before starting the incubation. After $2-12 \mathrm{~h}$ of culture cells were lysed in RLT buffer (Qiagen, Hilden, Germany) and total RNA was extracted. In order to measure secreted mediators and proteins, cells were cultured for $24 \mathrm{~h}$. Supernatants were then collected and stored at $-80{ }^{\circ} \mathrm{C}$ until use.

THP-1 cells (from Cell Lines Service Eppelheim, Germany) were maintained at $<2 \times 10^{5}$ cells $/ \mathrm{mL}$ in RPMI 1640 medium supplemented with $50 \mathrm{U} / \mathrm{mL}$ penicillin, $50 \mu \mathrm{g} / \mathrm{mL}$ streptomycin, $10 \%$ FCS and $2 \mathrm{mM}$ L-glutamine. Cells were treated with $50 \mathrm{nM}$ phorbol 12-myristate 13acetate (PMA) to induce adherence and differentiation into macrophages. After 2 days of culture, cells were incubated with RES and activated with LPS/IFN- $\gamma(100 \mathrm{ng} / \mathrm{mL}$ LPS, $20 \mathrm{U} / \mathrm{mL}$ IFN- $\gamma$ ). For gene expression analysis, total RNA was extracted from THP-1 cells $4 \mathrm{~h}$ after activation. Cell culture supernatants were recovered after $24 \mathrm{~h}$ and processed for chemokine and cytokine analysis.

HUVECs were from Lonza, (Walkersville, MD), cultured in EGM (Endothelial Growth Medium, Lonza) and used for experiments between passages 3 to 7. Cells $\left(2 \times 10^{5}\right.$ per well $)$ were seeded into BioCoat ${ }^{\mathrm{Tm}}$ Collagen I 6-well plates (Becton Dickinson, San Jose, CA). Cells were activated with TNF- $\alpha(10 \mathrm{ng} / \mathrm{mL})$ or IL-1 $\beta$ (5 ng/ $\mathrm{mL}$ ) and cultured for 2-24 h. All treatments were done in duplicate and all experimental series were done at least twice.

\section{RNA isolation, cDNA synthesis and quantitative RT-PCR}

The isolation of total RNA, synthesis of cDNA and quantitative RT-PCR has been performed and quantified as detailed before [9]. Sequences of primers and probes used for Taqman ${ }^{\text {Th }}$ gene expression analysis are given in Additional File 1. Results were obtained from triplicates and are indicated as fold changes ( \pm errors, calculated with a logarithmic function) (see [9]).

\section{Multiparametric analysis of cytokines, chemokines and interleukins}

Multiparametric kits for the quantification of chemokines, cytokines and interleukins were purchased from BIO-RAD Laboratories (Hercules, CA) and used in the LiquiChip Workstation IS 200 (Qiagen, Hilden, Germany). Data evaluation was made with the LiquiChip Analyser software (Qiagen) [10]. Nitric oxide (NO), which is quantified as the stable nitrite $\left(\mathrm{NO}_{2}\right)$ by the Griess reaction, and prostaglandin $\mathrm{E}_{2}\left(\mathrm{PGE}_{2}\right)$ were measured as described previously [8].

\section{Statistical analysis}

Data were evaluated with the statistical tools described previously $[9,10]$. A $p$ value $<0.05$ (calculated by using Student's t test or one-way ANOVA) was considered to reflect statistically significant differences. Where appropriate, 
the Tuckey post-hoc test was applied for multiple comparisons. Statistical analyses were performed with SPSS 23.0.0.0 (SPSS, Munich, Germany).

\section{Results}

Anti-inflammatory effects of RES in murine RAW 264.7

cells

Initially, we studied the influence of RES on the inflammatory profile of LPS-stimulated murine macrophages (RAW267.4 cells), which express numerous inflammatory genes [11]. RES reduced the production of nitric oxide (NO) and the secretion of COX-2 dependent prostaglandin $\mathrm{E}_{2}\left(\mathrm{PGE}_{2}\right)($ Table 1$)\left(\mathrm{IC}_{50}=27.7 \pm 1.6 \mu \mathrm{M}\right.$ $[n=24]$ and $19.0 \pm 2.4 \mu \mathrm{M}[n=20]$ for $\mathrm{NO}$ and $\mathrm{PGE}_{2}$, respectively). Furthermore, we analyzed the effects of RES on cytokine and chemokine (CK) secretion in LPSactivated macrophages. RES blunted secretion of IL-1 $\beta$, IL-6, IL-12(p70), and TNF- $\alpha$. At 25-50 $\mu \mathrm{M}$, it also significantly reduced production of CCL5/RANTES, but had only marginal effect on CCL2/MCP-1 and CCL4/MIP-1 $\beta$ (Fig. 1). Notably, RES also impaired LPS-induced GMCSF production in the murine macrophage cell line.

Next, we investigated whether the expression of inflammatory genes was influenced by RES in RAW264.7 cells. RES diminished mRNA levels of IL-1 $\alpha$, IL-1 $\beta$, IL-6, TNF- $\alpha$, CCL4/MIP- $1 \beta$ and CCL5/RANTES (Fig. 2). RES exerted significantly stronger effects at the transcriptional level as compared to protein secretion, since effects were observed at RES concentrations as low as $6.25 \mu \mathrm{M}$. RES also impaired the NF- $\mathrm{KB}$ transcription pathway, since it reduced the transcription level of NF$\kappa B 1$ (Table S1). This is consistent with data reported by Tsai et al. [12].

\section{RES altered the cytokine and chemokine profile in human} THP- 1 cells

Upon treatment with phorbol-myristate acetate (PMA), the human monocytic leukemia cell line THP-1 expressed macrophage features [13]. RES has been found to modulate various biochemical parameters of THP-1 cells [14-16]. In this study we analyzed the impact of RES on inflammatory mediators produced by in vitro differentiated THP-1 cells. When activated with LPS, THP-1 cells

Table 1 Production of $\mathrm{PGE}_{2}$ and nitric oxide in LPS-stimulated RAW264.7 cells

\begin{tabular}{llll}
\hline Metabolite & LPS alone & LPS + RES $(25 \mu \mathrm{M})$ & $p$ \\
\hline PGE $_{2}[\mathrm{pg} / \mathrm{mL}]$ & $6334 \pm 306$ & $2816 \pm 134$ & 0.010 \\
Nitric oxide $(\mu \mathrm{M})$ & $20.1 \pm 0.8$ & $12.1 \pm 0.4$ & 0.005
\end{tabular}

Cells were incubated with $25 \mu \mathrm{M}$ RES, stimulated with $1 \mu \mathrm{g} / \mathrm{mL}$ LPS and cultured for $24 \mathrm{~h}$. 'LPS alone': indicates the value obtained from LPS-stimulated cells (without RES). Results are shown as mean ( \pm SEM) of triplicate cultures obtained in 5 independent experimental series. $p$ values were calculated between treatments 'LPS-alone' versus 'LPS + RES'. Unstimulated cells produced metabolites at $<10 \%$ of LPS-stimulated cells up-regulated cytokine and chemokine genes (Fig. 3) and secreted large amounts of $\mathrm{PGE}_{2}$, cytokines and chemokines, although not as abundantly as RAW264.7 cells (Table 2). RES was a potent inhibitor of $\mathrm{PGE}_{2}$ production in THP-1 cells for which an $\mathrm{IC}_{50}$ value of $5.0 \pm 1.2 \mu \mathrm{M}$ $(N=5)$ was calculated. At $25 \mu \mathrm{M}$ RES almost completely blunted $\mathrm{PGE}_{2}$ production (Table 2). Also, IL-1 $\beta$, IL-6 and CXCL10/IP-10 were significantly decreased by $25 \mu \mathrm{M}$ RES. In LPS-activated THP-1 cells, RES significantly down-regulated gene expression levels of IL-1 $\beta$, IL-6, TNF- $\alpha$ and chemokines including CCL2/MCP-1 and CXCL10/IP-10 (Fig. 3). It should be noted that RES selectively altered expression of these chemokines, since others, like CXCL/8/IL-8, were not modified by the polyphenolic compound.

\section{RES impaired the inflammatory response of LPS-activated} PBLs

RES is rapidly absorbed from the diet and it reaches peak levels in the blood within 1-6 h after ingestion [17]. This prompted us to more extensively analyze the impact of RES on inflammatory parameters of blood cells. To this aim, human PBLs were stimulated with LPS/INF- $\gamma$ in the presence of graded amounts of RES and the inflammatory response was quantified by measuring secreted mediators and gene expression. RES concentration-dependently inhibited $\mathrm{PGE}_{2}$ production (Fig. 4 and Table 3). RES had equivocal effects on TNF- $\alpha$ secretion, since high concentrations reduced it, whereas low concentrations rather enhanced it.

With regard to CKs, RES (at $>12.5 \mu \mathrm{M}$ ) inhibited CCL2/MCP-1, CCL5/RANTES and CXCL/IL-8 (Fig. 4 and Table 3). On the other hand, RES stimulated the secretion of CCL4/MIP-1 $\beta$ and CXCL10/IP-10. The production of IL-1 $\beta$ and IL-6 was concentrationdependently augmented by RES; this is in agreement with previous results [9]. In addition, RES had an overall inhibitory effect on the cytokines and chemokines produced by un-activated PBLs, which secreted only a small fraction of mediators compared to those produced by LPS-stimulated PBLs (Additional file 1: Figure S1). It should be emphasized that cell viability was not impaired at all tested RES concentrations (Additional file 1: Figure S2). PBL monocytes produced detectable amounts of GM-CSF after LPS-activation. Different from the observations made with murine macrophages, RES (at $25 \mu \mathrm{M}$ ) did not impair GM-CSF production in activated PBLs from different donors (Table 3).

Next, we investigated inflammatory gene expression in human PBLs and studied the impact of RES during the early phase of the inflammatory response. PBLs responded to LPS stimulation by the expression of thousands of genes [11]. Inflammatory genes including IL-1 $\beta$, IL-6, TNF- $\alpha$ or CXCL8/IL-8 were markedly up-regulated 


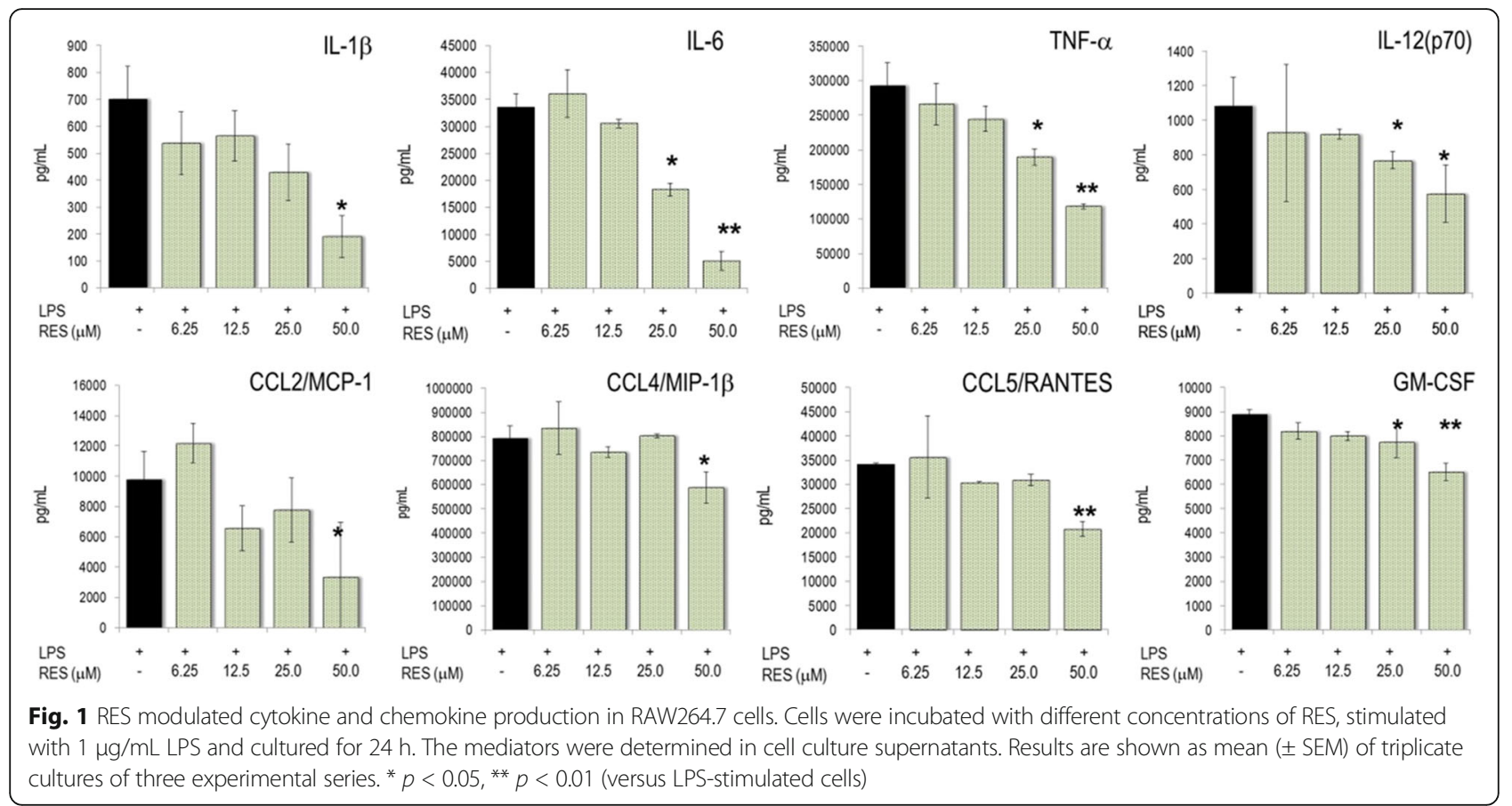

within $2 \mathrm{~h}$ of stimulation (Fig. 5) (see also [9]). RES drastically altered the expression levels of cytokines and chemokine genes, since TNF- $\alpha$ and CXCL8/IL-8 were significantly reduced. Conversely, it strongly up-regulated e.g. IL-6 and CXCL10/IP-10. IL-1 $\beta$ expression was not modified by RES. CCL2/MCP-1 mRNA levels were lower in $25 \mu \mathrm{M}$ RES-treated LPS-activated PBL. It should be emphasized that CCL5/RANTES gene expression was not induced by LPS, nor was it affected by RES (Fig. 5).

Many of LPS-induced genes in PBLs are regulated by transcription factors (TF). Accordingly, LPS up-regulated TFs of the NF-kB pathway and STAT. When PBLs were
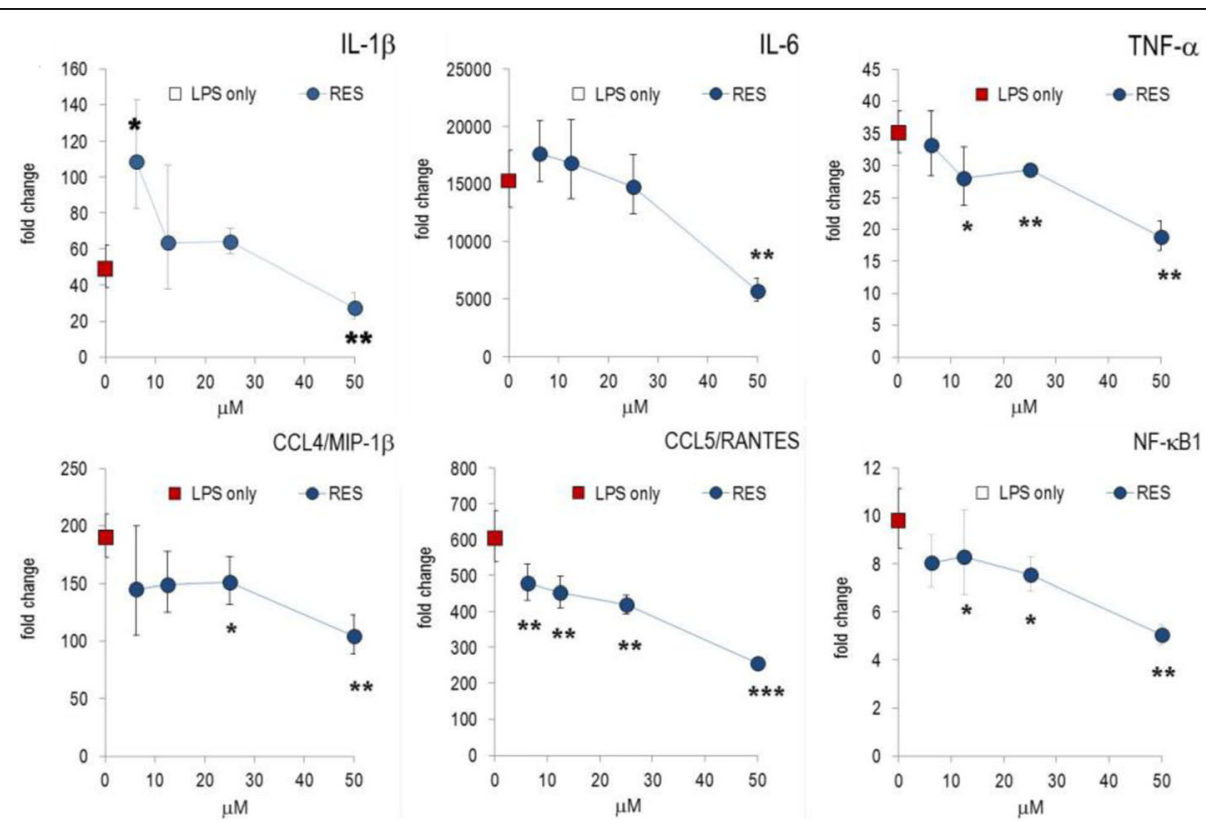

Fig. 2 RES modified gene expression in LPS-stimulated RAW264.7 cells. Cells were incubated with RES, and stimulated with $1 \mu \mathrm{gg} / \mathrm{mL}$ LPS and cultured for $4 \mathrm{~h}$. Gene expression was quantified by RT-PCR and the data expressed as fold change compared to levels observed in unstimulated cells. Results are shown as mean of triplicates ( \pm errors) (see reference [11]). 'LPS alone': indicates the value obtained from LPS-stimulated cells (without substance) and is indicated on the $y$-axis. ${ }^{*} p<0.05,{ }^{* *} p<0.01,{ }^{* * *} p<0.001$ (versus LPS-stimulated cells) 


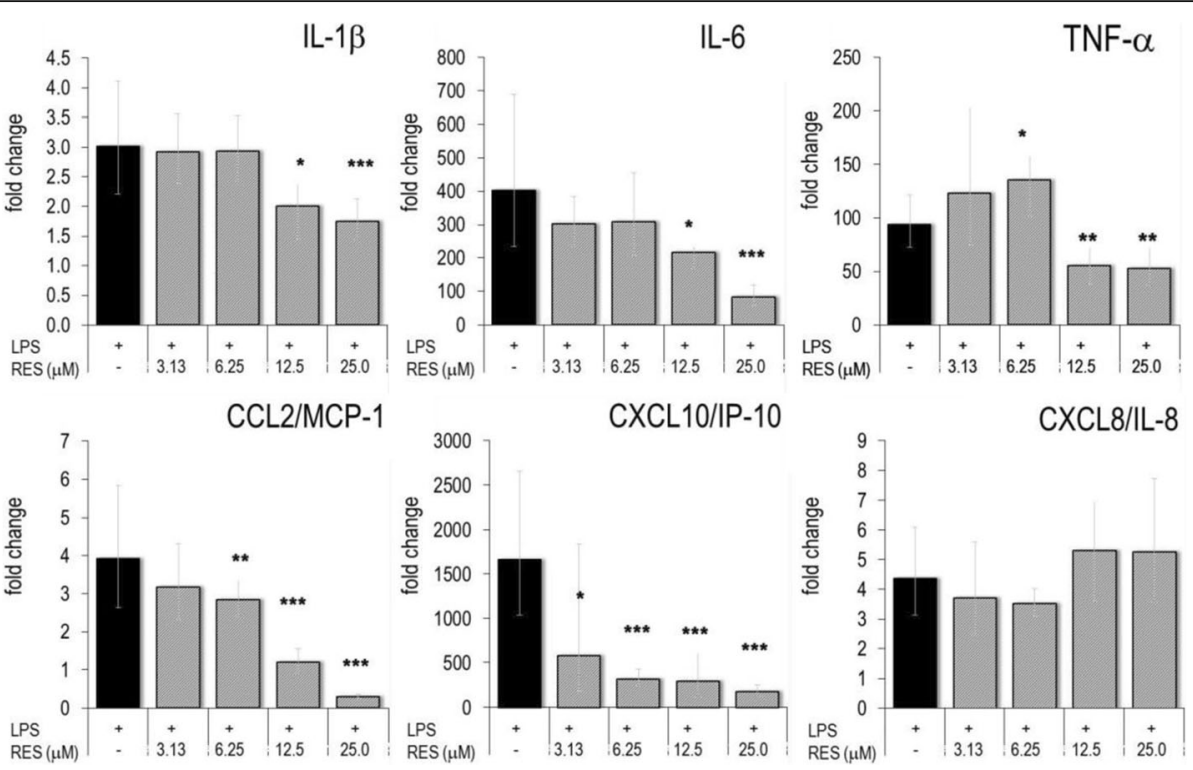

Fig. 3 Effect of RES on gene expression in activated THP-1. PMA-treated THP-1 cells were cultured in the presence of indicated concentrations of RES and activated with LPS for $4 \mathrm{~h}$. RT-PCR was performed and the gene expression levels were indicated as mean fold changes ( \pm errors) (versus unstimulated cell) (see also reference [11]). ${ }^{*} p<0.05,{ }^{* *} p<0.01,{ }^{* * *} p<0.001$ (versus LPS-stimulated cells)

activated in the presence of RES, mRNA levels of NF- $\mathrm{kB} 2$, STAT1 and CREB1 were reduced (Fig. 5). Collectively, the data indicate that RES modulated cytokine and chemokine expression at the transcriptional level via TFs.

\section{Modulation of inflammatory parameters associated with endothelial dysfunction}

Several studies previously showed that RES modified biological features of the vascular-endothelial compartment (see e.g. [18]). Therefore, we examined the effects of RES on inflammatory features of the endothelial cells. To this aim, we induced endothelial dysfunction (ED) by activating HUVECs with TNF- $\alpha$ or IL- $1 \beta$ and measured the impact of RES in these cells (Fig. 6). Stimulation of HUVECs with TNF- $\alpha$ induced an up to $~ 100$-fold increase of inflammatory mediators (see Legend to Fig. 6). RES significantly diminished the secretion of $\mathrm{PGE}_{2}$, IL-6, CCL2/MCP-1, and CXCL10/IP-10, whereas CCL5/ RANTES and CXCL8/IL-8 were resilient to RES treatment. Pro-inflammatory stimuli (i.e. TNF- $\alpha$ ) also induced the production of GM-CSF in HUVECs, where it promoted the differentiation of macrophages into foam cells [19]. GM-CSF production by TNF- $\alpha$ activated HUVECs was virtually abrogated by high concentrations of RES (Fig. 6). Unstimulated HUVECs secreted low amounts of metabolites (Additional file 1: Table S2).

TNF- $\alpha$ activation of HUVECs induced marked upregulation of gene expression of IL-6, ICAM-1, VCAM-1, CCL2/MCP-1, CXCL8/IL-8 and CXCL10/ IP-10 (Fig. 7). Most of these genes were concentrationdependently regulated by RES, which significantly decreased expression levels of IL-6 and CXCL10/IP-10. Unlike VCAM-1 expression, ICAM-1 gene expression was significantly reduced by RES. Moreover, RES reduced GM-CSF gene expression to levels observed with unstimulated cells. This is consistent with the features obtained at the level of secretion of inflammatory mediators. Another characteristic of ED induced by TNF- $\alpha$ is the down-regulation of endothelial nitric oxide synthase (eNOS) in HUVECs. Accordingly, TNF- $\alpha$ treatment diminished eNOS gene expression, while RES moved eNOS expression back to homeostatic levels (see also [20]).

\section{Discussion}

The biological activities of RES have been evaluated in a plethora of studies (for recent reviews see $[5-7,21]$ ).

Table 2 Resveratrol reduced the secretion of cytokines and chemokines in LPS-activated THP-1 cells

\begin{tabular}{|c|c|c|c|c|c|c|c|c|c|c|}
\hline \multirow[t]{2}{*}{ Treatment } & \multirow{2}{*}{$\begin{array}{l}\mathrm{PGE}_{2} \\
\mathrm{pg} / \mathrm{mL}\end{array}$} & \multirow[t]{2}{*}{$p$} & \multirow{2}{*}{$\begin{array}{l}\text { IL-1 } \\
\mathrm{pg} / \mathrm{mL}\end{array}$} & \multirow[t]{2}{*}{$p$} & \multirow{2}{*}{$\begin{array}{l}\text { IL-6 } \\
\mathrm{ng} / \mathrm{mL}\end{array}$} & \multirow[t]{2}{*}{$p$} & \multirow{2}{*}{$\begin{array}{l}\text { TNF-a } \\
\mathrm{pg} / \mathrm{mL}\end{array}$} & \multirow[t]{2}{*}{$p$} & \multicolumn{2}{|l|}{ CXCL10/IP-10 } \\
\hline & & & & & & & & & $\mathrm{pg} / \mathrm{mL}$ & $p$ \\
\hline LPS alone & $3010 \pm 333$ & & $4225 \pm 261$ & & $566 \pm 9$ & & $12,050 \pm 70$ & & $20,350 \pm 353$ & \\
\hline LPS + RES $(25 \mu \mathrm{M})$ & $515 \pm 65$ & 0.001 & $2095 \pm 21$ & 0.034 & $75 \pm 4$ & 0.013 & $11,750 \pm 495$ & 0.590 & $14,700 \pm 707$ & 0.028 \\
\hline
\end{tabular}

PMA-treated THP-1 cells were activated with LPS in the absence or presence of $25 \mu \mathrm{M}$ RES. The secreted metabolites were measured after $24 \mathrm{~h}$ of culture. Mean values $\pm \mathrm{SD}$ of triplicate cultures are shown. $p$ values were calculated between treatments 'LPS-alone' versus 'LPS + RES' 


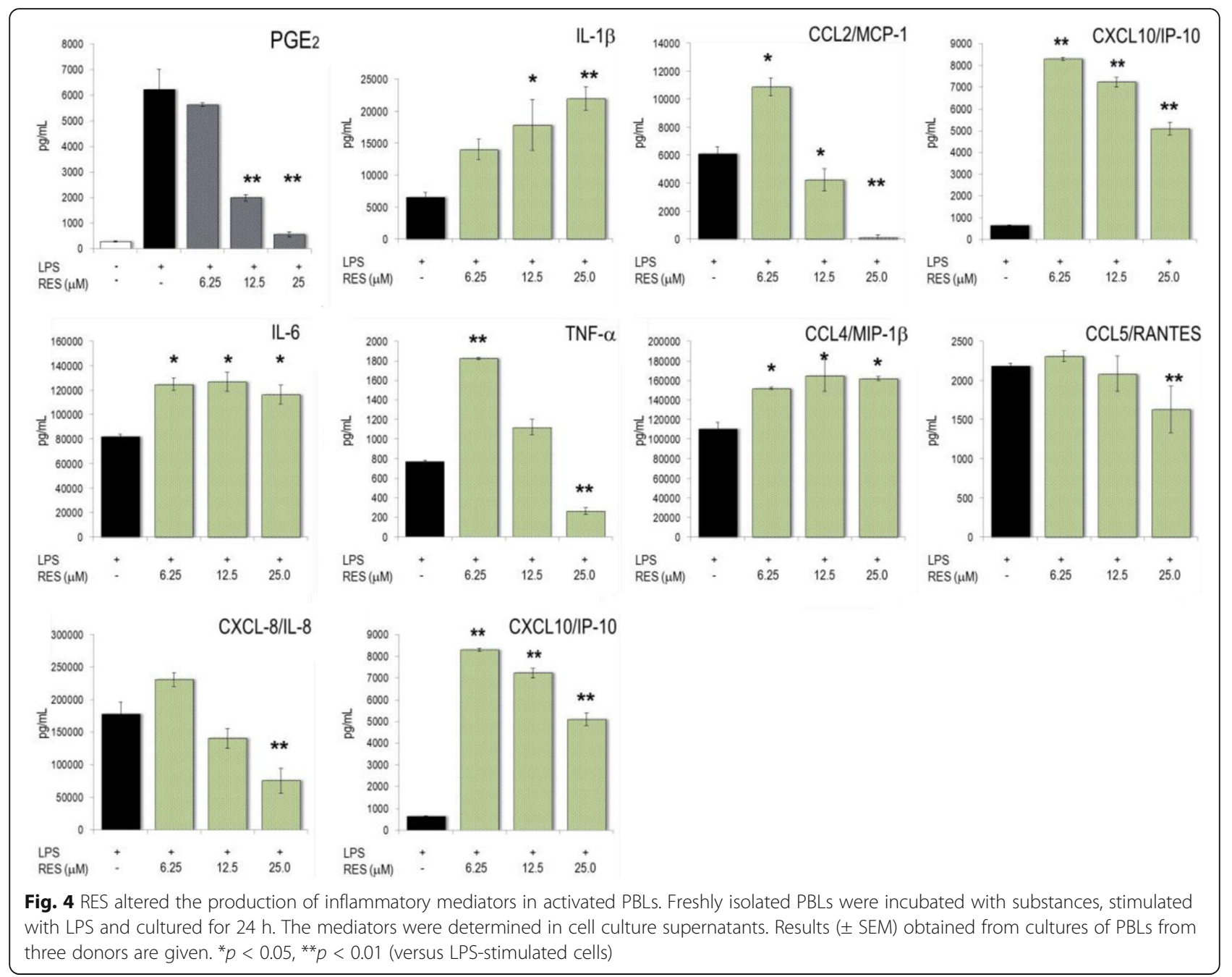

Table 3 RES-induced changes of the chemokine and cytokine profile in human PBLS

\begin{tabular}{llll}
\hline Metabolite & LPS/INF- $\gamma$ alone & RES $(25 \mu \mathrm{M})+$ LPS/INF- $\gamma$ & $p$ \\
\hline PGE $_{2}[\mathrm{pg} / \mathrm{mL}]$ & $4975 \pm 483$ & $612 \pm 238$ & 0.005 \\
IL-6 $[\mathrm{ng} / \mathrm{mL}]$ & $76.8 \pm 5.5$ & $135.1 \pm 16.7$ & 0.044 \\
IL-1 $\beta[\mathrm{ng} / \mathrm{mL}]$ & $11.6 \pm 1.3$ & $22.3 \pm 2.2$ & 0.009 \\
TNF-a $[\mathrm{pg} / \mathrm{mL}]$ & $2762 \pm 393$ & $1195 \pm 320$ & 0.04 \\
CCL2/MCP-1 $[\mathrm{pg} / \mathrm{mL}]$ & $804 \pm 60$ & $352 \pm 26$ & 0.002 \\
CCL4/MIP-1 $\beta[\mathrm{ng} / \mathrm{mL}]$ & $70.8 \pm 6.2$ & $123 \pm 22$ & 0.16 \\
CCL5/RANTES $[\mathrm{pg} / \mathrm{mL}]$ & $1032 \pm 220$ & $798 \pm 59$ & 0.05 \\
CXCL10/IP-10 [pg/mL] & $2072 \pm 110$ & $16,758 \pm 2649$ & 0.026 \\
GM-CSF & $17.9 \pm 3.3$ & $15.2 \pm 3.0$ & 0.29 \\
\hline
\end{tabular}

Freshly isolated PBLs were stimulated with LPS/INF- $\gamma$ in the presence of RES and cultured for $24 \mathrm{~h}$. Metabolites were determined in the culture supernatants by multiplex ELISA. Mean $( \pm S D)$ of triplicate cultures from three donors are shown. $p$ values were calculated between treatments 'LPS/INF- $\gamma$ -alone' versus 'RES + LPS/INF- $\gamma^{\prime}$
Hence, RES becomes one of the most intensely examined plant secondary metabolite ever. Not unexpectedly, many controversies have grown [6], since the effects of RES were pleiotropic and relied on diverse mode of actions in different cells, tissue, metabolic states and species [21]. In this study we compared the effects of RES on the production of chemokines and cytokines in different cell types, which represent the innate immune system (i.e. macrophages) and the vascular-endothelial compartment (i.e. PBLs and HUVECs, respectively). The observed data require a revisited holistic view of the polyphenolic substance, in which the various features of RES are connected with their temporal and systemic occurrence in the inflammatory and immune response (Fig. 8).

During the body's response to homeostatic changes due to external and internal causes, a large cascade of metabolic and cellular events occurs and reveals the status of inflammation. The subsequent cell migration during the innate and adaptive immune response is orchestrated and 


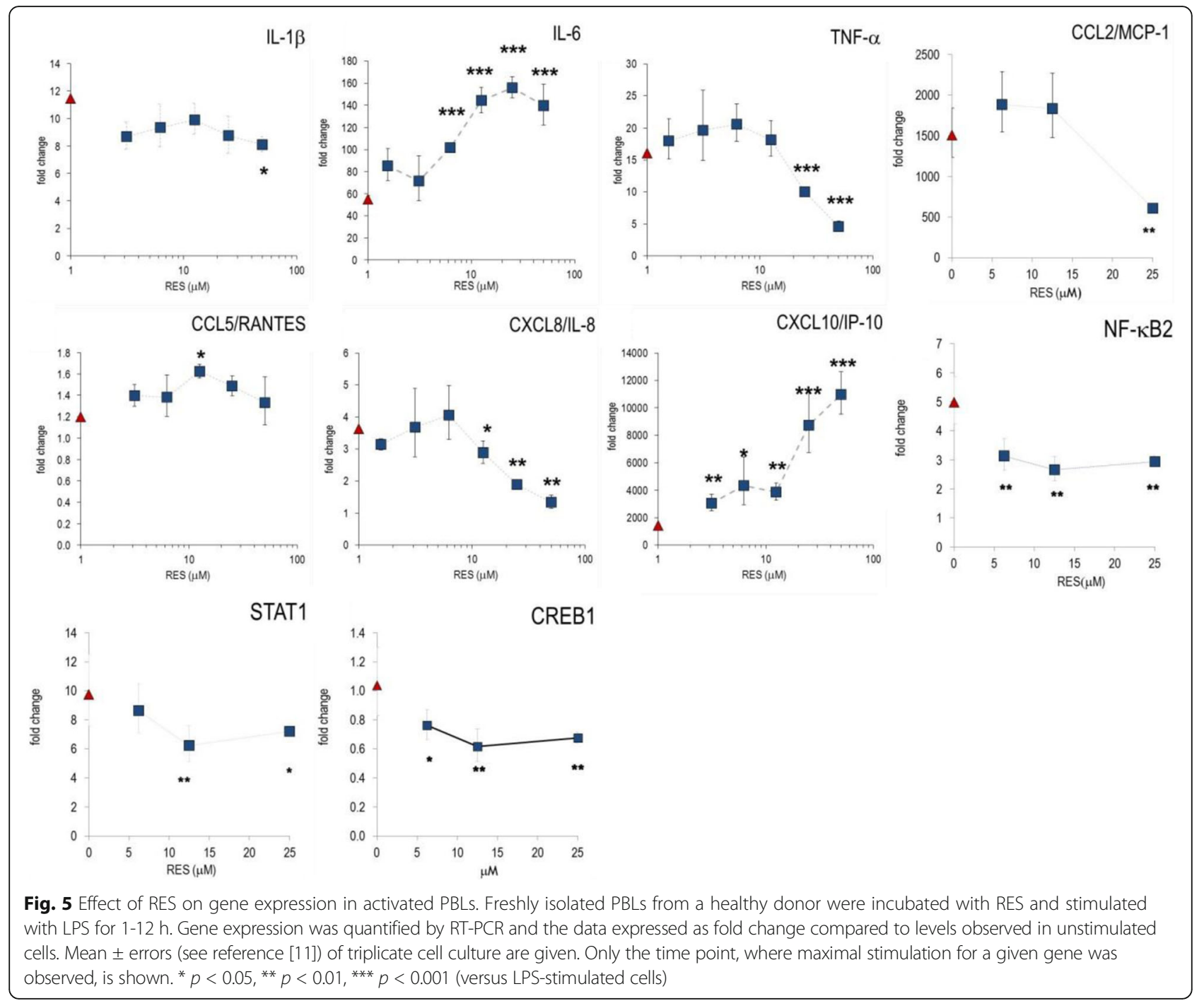

controlled by cytokines and CKs. CCL2/MCP-1, CCL3/ MIP- $1 \alpha$ and CCL4/MIP-1 $\beta$ recruit monocytes. CCL5/ RANTES and CXCL10/IP-10 enable chemotaxis of activated $\mathrm{T}$ lymphocytes. Neutrophils are recruited by CXCL8/IL-8 [22]. A major and novel feature of the present study relies on the observation that RES cellspecifically modulated the chemokine production; in the blood compartment (i.e. PBLs), RES dampened the production of CCL5/RANTES and CXCL8/IL-8, but enhanced CCL4/MIP-1 $\beta$ and CXCL10/IP-10 (Fig. 4). Subpopulations of PBLs such as lymphocytes, monocytes/macrophages and neutrophils responded to LPS at various degrees [9]. Presumably, RES has genuine effects on each of these populations. By altering the delicate balance between CKs, RES might influence the temporal pattern of cell migration during the different phases of the innate immune response. Surprisingly, RES was found to enhance the expression of IL-1 $\beta$ and
IL-6 in the peripheral compartment but not in macrophages or in the endothelium (see below).

Upon TNF- $\alpha$ stimulation HUVECs expressed a pattern of cytokines and chemokines, which had many common features with that of LPS-activated PBL. In endothelial cells, RES corrected CK expression in a comparable way as in PBL, although the extent of changes was less striking. RES also reduced the expression of ICAM-1 towards pre-ED homeostasis, whereas VCAM-1 expression was insignificantly influenced by the polyphenol. Thus, a diminished endothelial diapedesis might be one biological consequence of the presence of RES in the blood. Our data are in agreement with other studies, which showed that RES down-regulated ICAM-1 and mitigated chemotaxis and vascular inflammation via CCL2/MCP-1 and NF- $\mathrm{B}$, respectively [23]. The pleiotropic effects of RES in HUVECs were further underscored by the RESdependent increase of eNOS expression at ED conditions 

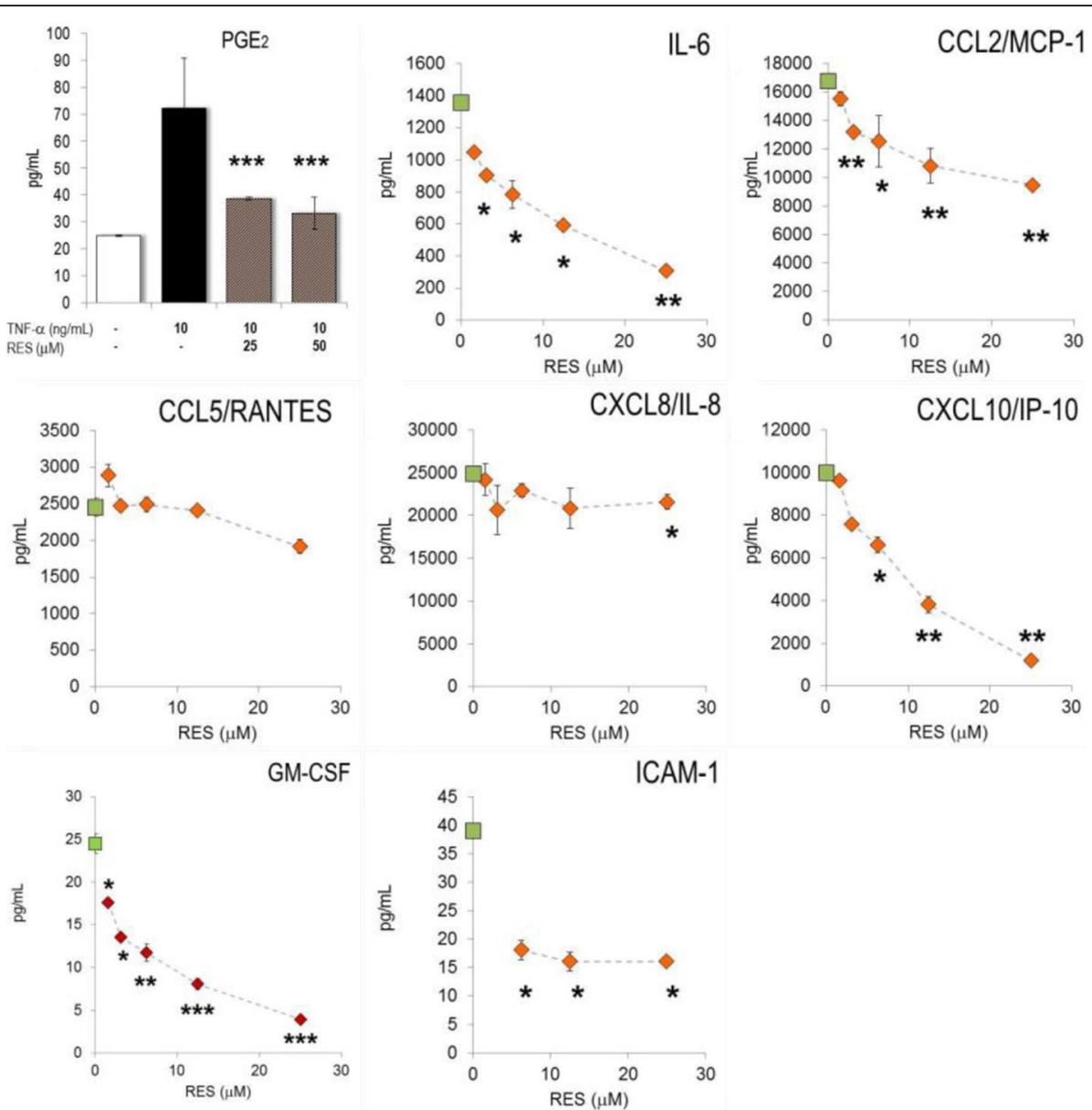

Fig. 6 Secretion of mediators by activated HUVECs. Cells were incubated with RES, activated with $10 \mathrm{ng} / \mathrm{mL}$ TNF-a and cultured for $24 \mathrm{~h}$. The secreted cytokines, chemokines, ICAM-1 and VCAM-1 were quantified by Luminex technology (see Methods). Mean \pm SD of triplicates are given. Unstimulated cells produced $<10 \%$ of the mediators secreted by TNF-a stimulated cells. ${ }^{*} p<0.05,{ }^{* *} p<0.01{ }^{* * *} p<0.001$ (versus TNF-a -stimulated cells)

$[20,23,24]$. Notably, the production of GM-CSF, a key marker of atheroma formation $[19,25]$ and for the differentiation of pro-inflammatory macrophages [26], was robustly blunted by the presence of RES. Collectively, the plant metabolite was shown to improve endothelial functions at various levels. Chronic in vivo exposure of dys-regulated endothelium to RES is expected to beneficially modulate vascular function.

Macrophages, which develop from monocytes, are pivotal during initiation and resolution of inflammation and they differentiate into tissue-specific subsets [27]. Nitric oxide $(\mathrm{NO})$ and $\mathrm{PGE}_{2}$ are hallmarks of both acute and chronic inflammation in pro-inflammatory macrophages. RES impaired iNOS-dependent NO production in RAW264.7 macrophages (Fig. 1 and [12] and exerted strong inhibitory effects on $\mathrm{PGE}_{2}$ and thus clearly met the criteria of an anti-inflammatory nutrient. Likewise, the production and expression of cytokines including TNF- $\alpha$ and the pro-inflammatory IL- $1 \beta$ and IL- 6 were impaired by RES, suggesting that it dampened the classical activation of macrophages. With regard to CKs, RES blunted CCL4/MIP-1 $\beta$ and CCL5/RANTES and therefore the recruitment of monocyte and activated $\mathrm{T}$ lymphocytes, respectively. Notably GM-CSF, a growth and differentiation factor for pro-inflammatory macrophages, was robustly diminished by RES. These effects were not species-specific, since analogous results were observed with the human monocytic leukemia cell line THP-1. RES altered cell morphology, gene expression, ligand-receptor interactions, signaling pathways and eventually foam-cell formation [14-16, 28]. Collectively, RES had an overall anti-inflammatory profile in macrophages. This is consistent with observations in chondrocytes at conditions that mimic chronic inflammation associated with osteoarthritis [33].

The molecular regulation of inflammatory processes is critically governed by transcription factors (TF). LPSactivation of monocytes and macrophages induced the 


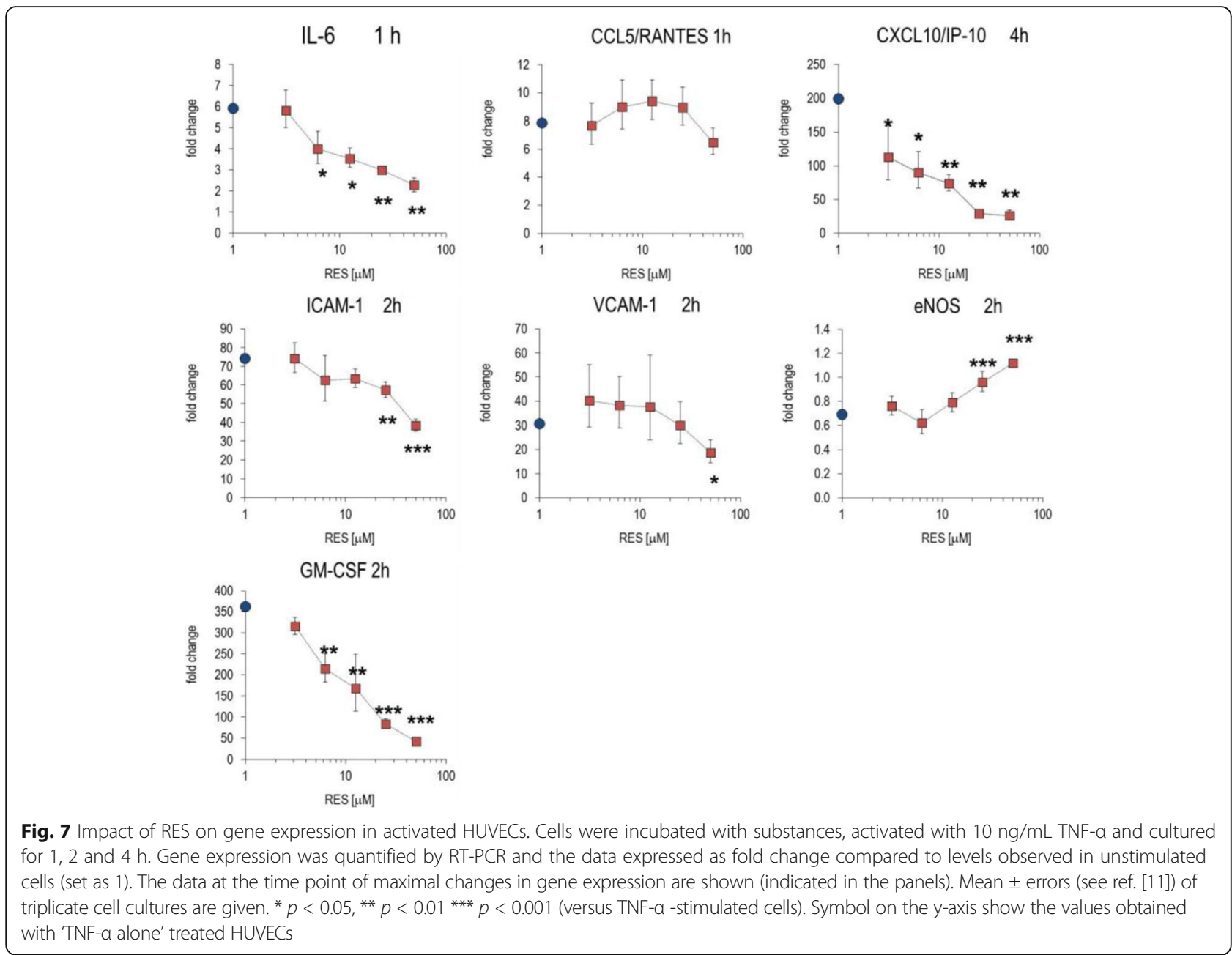

NF- $\mathrm{KB}$ dependent transcription of chemokines such as CXCL8/IL-8, CXCL10/IP-10, CCL2/MCP1 and CCL5/ RANTES [29-31]. As shown in this study, RES influenced the transcription level of NF- $\kappa B$ elements as well as STAT1 and CREB1 (for reviews see [5, 32]). Likewise, TNF- $\alpha$ induced activation of the NF- $\kappa B$ elements is modulated by RES and its related substance piceatannol. Further investigations should evaluate whether RES has distinct effects on polymorphonuclear cells, including neutrophils, monocytes/macrophages or lymphocytes.

The idiosyncratic effects of RES in three interconnected cellular systems are summarized in Fig. 8. Except for the peripheral blood compartment, cytokines and chemokine markers are consistently altered in the same direction thus corroborating the favorable properties of RES on attenuating chronic or low-grade inflammation. Notably, $\mathrm{PGE}_{2}$ was strongly and consistently blunted in PBLs, macrophages endothelial cells and chondrocytes [9,33]. Given the widespread importance of $\mathrm{PGE}_{2}$ in the regulation of the immune response [34], RES appears to predominantly modulate the immune response by influencing cellular
$\mathrm{PGE}_{2}$ levels. It should be noted that RES did not significantly change COX-2 gene expression in various cellular systems $[9,33]$ and hence does not impair the COX2 dependent production of mediators required during the resolution of inflammation [35]. The cell-specific effect on interleukin production is another salient feature of RES, since it favored production of IL-1 $\beta$ and IL-6 in PBLs, but it had opposite effects in macrophages. Enhanced production of IL-1 $\beta$ and IL- 6 is considered to characterize a pro-inflammatory status, but it is also involved in tissue regeneration [36]. In the immune response, however, IL- $1 \beta$ and IL- 6 contribute to $T_{h}$ lymphocyte differentiation and function $[37,38]$. Consequently, immune cells exposed to RES in the vascular compartment would produce higher levels of IL$1 \beta$ or IL- 6 and thus be primed for the adaptive immune response. Since RES has a short half-life in the blood [39], it exerts peripheral effects on immune cells only for a limited time. The RES-induced enhancement of immune responsiveness by IL- $1 \beta$ or IL- 6 is high in blood cells, whereas it is reverted in macrophages, which are key 


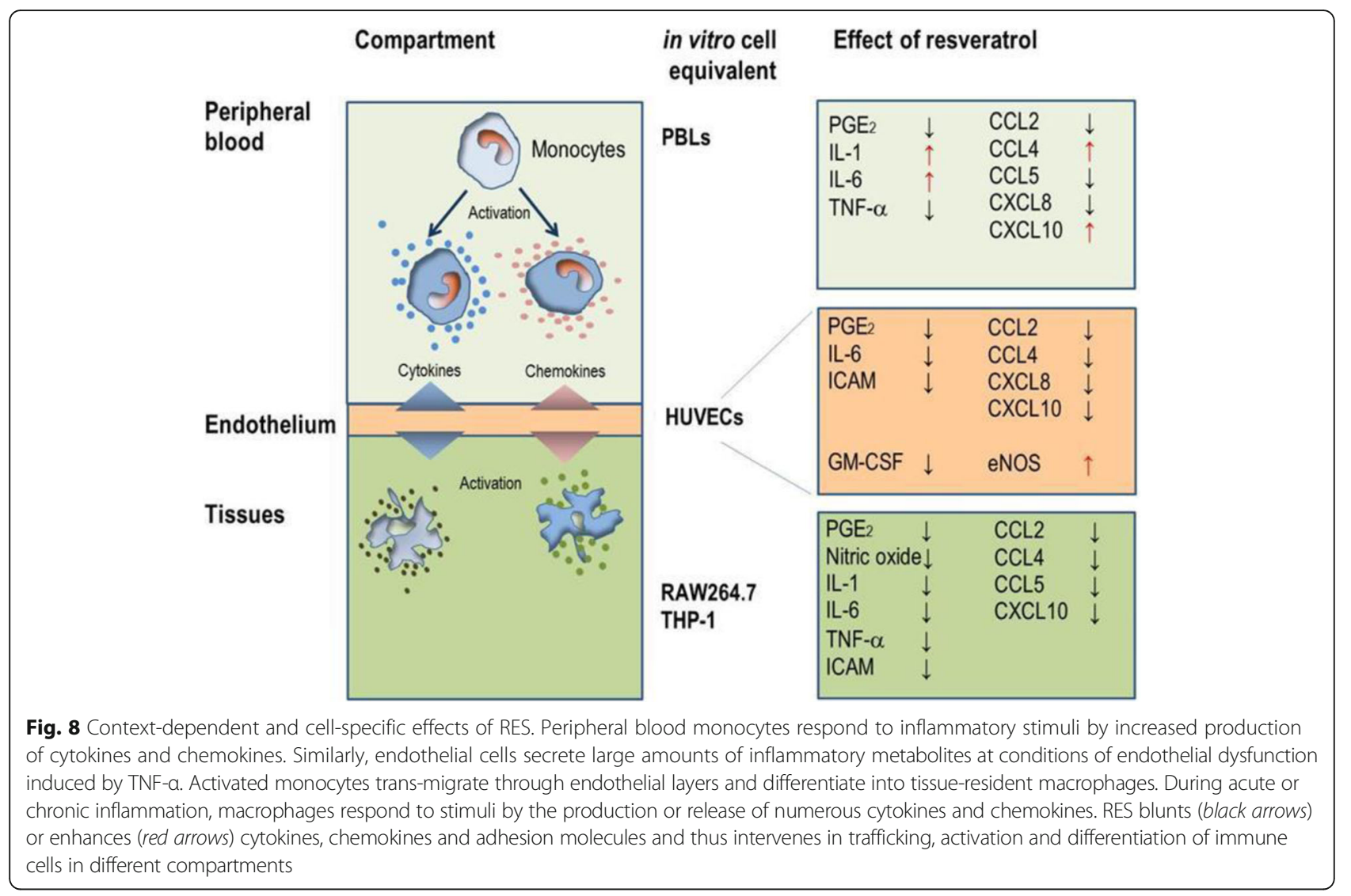

players in low-grade and chronic inflammation. Hence RES contributes to improve the systemic response to 'danger' signals $[3,40]$ in the periphery and concomitantly reduce the low-grade inflammatory status related to chronic diseases in tissues. This would account for genuine and even opposite effects of RES in different biological contexts.

The diverse features of RES demonstrated in cellular systems need to be further evaluated in appropriate nutritional intervention studies. Bakker et al. supplemented overweight subjects for 5 weeks with an 'anti-inflammatory diet mixture' (AIM) comprising RES and observed subtle changes in expression levels of IL-12, ICAM-1 and VCAM-1, whereas AIM did not alter in vivo levels of CCL2/MCP-1, CCL3/MIP-1 $\alpha$, CCL5/RANTES, CXCL8/ IL-8 or $\mathrm{PGE}_{2}$ [4]. It is likely that nutrient-induced changes were no longer sensed during long supplementation periods and thus not measurable. The experimental set-up of the cellular study reflects features of short-term supplementation and thus acute inflammation. As a consequence the effects of nutrients on plasma metabolites or leukocyte gene expression will only be detected in vivo at conditions of immediately perturbed homeostasis as it is observed during food intake. In general, the observed effects required rather high RES concentrations, which are not met in plasma or only for a short period of time after food intake. Yet, it should be noted that in cellular and tissue compartments RES reached substantially larger levels [41].

\section{Conclusions}

This study shows that resveratrol modulates many mediators of the inflammatory response. Its effects are context-dependent, i.e. RES might influence chemokines and cytokines in opposite ways in different tissues. While we corroborate the overall anti-inflammatory effect of the polyphenol, we nuance it along a biological divide where beneficial effects might also imply an enhancement of a pro-inflammatory property which is required to rapidly respond to, and resolve, an acute inflammation.

\section{Additional file}

Additional file 1: Supplementary Information. (DOCX 117 kb)

\section{Abbreviations}

CCL: CC chemokine ligand; CK: chemokines; CXCL: CXC chemokine ligand; GM-CSF: Granulocyte-macrophage colony-stimulating factor; HUVECs: Human umbilical vein endothelial cells; IL: Interleukin; LPS: Lipopolysaccharide; NO: Nitric oxide; PBLs: Peripheral blood leukocytes; PGE 2 : Prostaglandin $E_{2}$; RES: Resveratrol 


\section{Acknowledgments}

We thank Albine Bompard for providing additional data with THP-1 cells, Silvia Manz for valuable technical assistance in RT-PCR and Giorgio la Fata for insightful comments on the manuscript. We thank Franz Roos and Caroline Mittler for advice and assistance in statistical analyses.

\section{Funding}

The publication costs for this study was funded by DSM Nutritional Products Ltd.

\section{Availability of data and materials}

The materials used in this study can be requested from J.S.

(jpschwager@gmail.com). The study is not based on genomics or proteomics data set that would require to be deposited in publicly available repositories.

\section{Authors' contributions}

JS conceived the study; FW and NR planned and performed the experimental work and compiled the data; IS analyzed the data, interpreted them and wrote the paper. All authors read and approved the final manuscript.

\section{Competing interests}

The authors are employees of DSM Nutritional Products, which

commercializes resveratrol as resVida ${ }^{\oplus}$.

\section{Consent to publish}

Not applicable: The study does not contain any individual persons' data.

\section{Ethics approval and consent to participate}

Human primary cell protocols were approved by the Swiss Federal Office of Public Health (No. A050573/2 to J. Schwager).

\section{Publisher's Note}

Springer Nature remains neutral with regard to jurisdictional claims in published maps and institutional affiliations.

Received: 23 December 2016 Accepted: 6 June 2017

Published online: 13 June 2017

\section{References}

1. Hotamisligil GS, Erbay E. Nutrient sensing and inflammation in metabolic diseases. Nature Rev Immunology. 2008;8:923-34.

2. Serhan CN, Savill J. Resolution of inflammation: the beginning programs the end. Nat Immunol. 2005;6:1191-7.

3. Chawla A, Nguyen KD, Goh YP. Macrophage-mediated inflammation in metabolic disease. Nature Rev Immunology. 2011;11:738-49.

4. Bakker GC, van Erk MJ, Pellis $L$, Wopereis $\mathrm{S}$, et al. An antiinflammatory dietary mix modulates inflammation and oxidative and metabolic stress in overweight men: a nutrigenomics approach. Am J Clin Nutr. 2010;91:1044-59.

5. Alcarón de la Lasta C, Villegas I. Resveratrol as an anti-inflammatory and anti-aging agent: mechanisms and clinical implications. Mol Nutr Food Res. 2005;49:405-30.

6. Bitterman JL, Chung JH. Metabolic effects of resveratrol: addressing the controversies. Cell Mol Life Sci. 2015;72:1473-88.

7. Raederstorff D, Kunz I, Schwager J. Resveratrol, from experimental data to nutritional evidence: the emergence of a new food ingredient. Ann N Y Acad Sci. 2013:1290:136-41.

8. Richard N, Arnol S, Hoeller U, Kilpert C, Schwager J. Hydroxytyrosol is the major anti-inflammatory compound in aqueous olive extracts and impairs cytokine and chemokine production in macrophages. Planta Med. 2011:77:1890-7.

9. Richard N, Porath D, Radspieler A, Schwager J. Effects of resveratrol, piceatannol, tri-acetoxystilbene, and genistein on the inflammatory response of human peripheral blood leukocytes. Mol Nutr Food Res. 2005:49:431-42

10. Schwager J, Hoeller U, Wolfram S, Richard N. Rose hip and its constituent galactolipids confer cartilage protection by modulating cytokine, and chemokine expression. BMC Complement Altern Med. 2011:11:105.

11. Takao K, Miyakawa T. Genomic responses in mouse models greatly mimic human inflammatory diseases. Proc Natl Acad Sci U S A. 2015:112:1167-72.

12. Tsai SH, Lin-Shiau SY, Lin JK. Suppression of nitric oxide synthase and the down-regulation of the activation of NFkappaB in macrophages by resveratrol. Br J Pharmacol. 1999;126:673-80.
13. Auwerx J. The human leukemia cell line, THP-1: a multifacetted model for the study of monocyte-macrophage differentiation. Experientia. 1991;47:22-31.

14. Cullen JP, Morrow D, Jin Y, von Offenberg SN, et al. Resveratrol inhibits expression and binding activity of the monocyte chemotactic protein-1 receptor, CCR2, on THP-1 monocytes. Atherosclerosis. 2007;195:e125-33.

15. Oh YC, Kang OH, Choi JG, Chae HS, et al. Anti-inflammatory effect of resveratrol by inhibition of IL-8 production in LPS-induced THP-1 cells. The American Journal of Chinese Medicine. 2009:37:1203-14.

16. Tsan MF, White JE, Maheshwari JG, Bremner TA, Sacco J. Resveratrol induces Fas signalling-independent apoptosis in THP-1 human monocytic leukaemia cells. Br J Haematol. 2000;109:405-12.

17. Marier JF, Vachon P, Gritsas A, Zhang J, et al. Metabolism and disposition of resveratrol in rats: extent of absorption, glucuronidation, and enterohepatic recirculation evidenced by a linked-rat model. J Pharmacol Exp Ther. 2002;302:369-73.

18. Pendurthi UR, Williams JT, Rao LV. Resveratrol, a polyphenolic compound found in wine, inhibits tissue factor expression in vascular cells: a possible mechanism for the cardiovascular benefits associated with moderate consumption of wine. Arterioscler Thromb Vasc Biol. 1999;19:419-26.

19. Lenhoff S, Olofsson T. Cytokine regulation of GM-CSF and G-CSF secretion by human umbilical cord vein endothelial cells (HUVEC) Cytokine. 1996:8:702-9.

20. Wallerath $\mathrm{T}$, Deckert $\mathrm{G}$, Ternes $\mathrm{T}$, Anderson $\mathrm{H}$, et al. Resveratrol, a polyphenolic phytoalexin present in red wine, enhances expression and activity of endothelial nitric oxide synthase. Circulation. 2002;106:1652-8.

21. Kulkarni SS, Canto C. The molecular targets of resveratrol. Biochim Biophys Acta. 1852:2015:1114-23.

22. Phillipson M, Kubes $P$. The neutrophil in vascular inflammation. Nat Med. 2011:17:1381-90.

23. Csiszar A, Smith K, Labinskyy N, Orosz Z, et al. Resveratrol attenuates TNF-alpha-induced activation of coronary arterial endothelial cells: role of NF-kappaB inhibition. American journal of physiology. Heart and Circulatory Physiology. 2006:291:H1694-9.

24. Pellegatta F, Bertelli AA, Staels B, Duhem C, et al. Different short- and long-term effects of resveratrol on nuclear factor-kappaB phosphorylation and nuclear appearance in human endothelial cells. Am J Clin Nutr. 2003;77:1220-8.

25. Fossati G, Mazzucchelli I, Gritti D, Ricevuti G, et al. In vitro effects of GM-CSF on mature peripheral blood neutrophils. Int J Mol Med. 1998;1:943-51.

26. Murray PJ, Allen JE, Biswas SK, Fisher EA, et al. Macrophage activation and polarization: nomenclature and experimental guidelines. Immunity. 2014:41:14-20.

27. Gordon S, Taylor PR. Monocyte and macrophage heterogeneity. Nature Rev Immunology. 2005:5:953-64.

28. Dong W, Wang X, Bi S, Pan Z, et al. Inhibitory effects of resveratrol on foam cell formation are mediated through monocyte chemotactic protein-1 and lipid metabolism-related proteins. Int J Mol Med. 2014;33:1161-8.

29. Richmond A. NF-kappa B, chemokine gene transcription and tumour growth. Nature Rev Immunology. 2002;2:664-74.

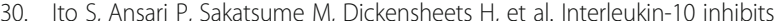
expression of both interferon alpha- and interferon gamma- induced genes by suppressing tyrosine phosphorylation of STAT1. Blood. 1999;93:1456-63.

31. Ohmori $Y$, Hamilton TA. Requirement for STAT1 in LPS-induced gene expression in macrophages. J Leukoc Biol. 2001;69:598-604.

32. Whitlock NC, Baek SJ. The anticancer effects of resveratrol: modulation of transcription factors. Nutr Cancer. 2012;64:493-502.

33. Schwager J, Richard N, Riegger C, Salem, N Jr. omega-3 PUFAs and Resveratrol Differently Modulate Acute and Chronic Inflammatory Processes. BioMed Res Int. 2015. doi:10.1155/2015/535189.

34. Kalinski P. Regulation of immune responses by prostaglandin E2. J Immunol. 2012;188:21-8.

35. Willoughby DA, Moore AR, Colville-Nash PR, Gilroy D. Resolution of inflammation. Int J Immunopharmacol. 2000:22:1131-5.

36. Karin $\mathrm{M}$, Clevers $\mathrm{H}$. Reparative inflammation takes charge of tissue regeneration. Nature. 2016:529:307-15.

37. Diehl S, Rincon M. The two faces of IL-6 on Th1/Th2 differentiation. Mol Immunol. 2002:39:531-6.

38. Mauer J, Chaurasia B, Goldau J, Vogt MC, et al. Signaling by IL-6 promotes alternative activation of macrophages to limit endotoxemia and obesityassociated resistance to insulin. Nat Immunol. 2014;15:423-30. 
39. Walle T, Hsieh F, DeLegge MH, Oatis JE Jr, Walle UK. High absorption but very low bioavailability of oral resveratrol in humans. Drug Metab Dispos. 2004:32:1377-82.

40. Gallucci S, Matzinger P. Danger signals: SOS to the immune system. Curr Opin Immunol. 2001;13:114-9.

41. Almeida L, Vaz-da-Silva M, Falcao A, Soares E, et al. Pharmacokinetic and safety profile of trans-resveratrol in a rising multiple-dose study in healthy volunteers. Mol Nutr Food Res. 2009;53(Suppl 1):S7-15.

Submit your next manuscript to BioMed Central and we will help you at every step:

- We accept pre-submission inquiries

- Our selector tool helps you to find the most relevant journal

- We provide round the clock customer support

- Convenient online submission

- Thorough peer review

- Inclusion in PubMed and all major indexing services

- Maximum visibility for your research

Submit your manuscript at www.biomedcentral.com/submit 